\title{
Whole genomes reveal multiple candidate genes and pathways involved in the immune response of dolphins to a highly infectious viral disease
}

\author{
Kimberley Batley ${ }^{1}$, Jonathan Sandoval-Castillo${ }^{1}$, Catherine Kemper ${ }^{2}$, Nikki Zanardo , \\ Ikuko Tomo ${ }^{2}$, Luciano Beheregaray ${ }^{1}$, and Luciana Moller ${ }^{1}$ \\ ${ }^{1}$ Flinders University \\ ${ }^{2}$ South Australian Museum
}

November 3, 2020

\begin{abstract}
Wildlife species are challenged and threatened by various infectious diseases that act as important selective forces and demographic drivers of populations. Yet, studies about host genetic factors and disease susceptibility are very limited. Cetacean morbillivirus (CeMV) has emerged as a major viral threat to cetacean populations worldwide, contributing to the death of tens of thousands of individuals of multiple dolphin and whale species. To understand the genomic basis of immune responses to CeMV, we generated and analysed whole genomes of 53 Indo-Pacific bottlenose dolphins (Tursiops aduncus) exposed to Australia's largest CeMV-related mortality event known to date. The genomic dataset consisted of 7,720,686 SNPs anchored onto 23 chromosome-length scaffolds and 77 short scaffolds. Allele frequency estimates between survivors and non-survivors of the outbreak revealed 11,009 candidate SNPs, of which 498 were annotated to 220 protein coding genes. These included 36 genes with functions related to innate and adaptive immune responses, and cytokine signalling pathways. The list also included genes known to be involved in immune responses to other morbilliviruses, such as measles in humans and the phocine distemper virus in pinnipeds. Our study characterised genomic regions and pathways that likely contribute to CeMV susceptibility and resistance in dolphins, representing a stride towards clarifying the complex interactions of the cetacean immune system. It also emphasises the relevance of whole genome datasets to study the genetics of wildlife diseases.
\end{abstract}

\section{Hosted file}

Batley et al. Main Document.pdf available at https://authorea.com/users/372402/articles/ 490457-whole-genomes-reveal-multiple-candidate-genes-and-pathways-involved-in-theimmune-response-of-dolphins-to-a-highly-infectious-viral-disease 